\title{
Templated Assembly of Block Copolymer toward Nonequilibrium Nanostructures in Thin Films
}

\author{
Shih-Huang Tung ${ }^{\dagger}$ and Ting $\mathrm{Xu}^{*, \dagger, *, 8}$ \\ ${ }^{\dagger}$ Department of Materials Science and Engineering and ${ }^{\star}$ Department of Chemistry, University of California, \\ Berkeley, California 94720-1760, and ${ }^{\S}$ Material Sciences Division, Lawrence Berkeley National Laboratory, \\ Berkeley, California 94720-1760
}

Received March 7, 2009; Revised Manuscript Received May 15, 2009

\begin{abstract}
We report a simple route to generate nonequilibrium nanostructures combining two known block copolymer (BCP) morphologies by first templating the spatial arrangement of BCP in thin films using a supramolecule. The BCP subsequently assembles within the morphological framework established by the supramolecule, leading to a templated, nonequilibrium nanostructures not accessible by the BCP alone. Thin films with hexagonally packed cylindrical domains oriented normal to the surface were formed initially by the self-assembly of the diblock copolymer-based supramolecules, comprised of symmetric polystyrene- $b$-poly(4-vinylpyridine) (PS- $b$-P4VP) with 3-pentadecylphenol (PDP) hydrogen-bonded to the 4VP. After selective removal of $\sim 90 \%$ of the PDP and a brief solvent annealing in a chloroform atmosphere, symmetric PS$b$-P4VP, containing a trace amount of PDP, self-assembled forming polygonal (dominantly hexagonal) microdomains oriented normal to the surface. This process reported should be applicable to the large library of copolymer-based supramolecules and enables the generation of novel nonequilibrium nanostructured morphologies. It also provides a new platform to study the pathway-dependent self-assembly in polymer thin films.
\end{abstract}

\section{Introduction}

Block copolymer (BCP) thin films are useful as templates and scaffolds for the fabrication of nanodevices. Controlling the BCP morphologies is critical, and as such, the phase behavior of BCP in thin films has attracted significant attention recently. ${ }^{1-11}$ In thin films, BCPs mainly adopt their bulk morphologies. The interfacial interactions of the blocks with the underlying substrate, ${ }^{12,13}$ the surface energies of the blocks, and the commensurability between the film thickness and the BCP equilibrium periodicity $^{14,15}$ dictate the BCP morphology and orientation of the microdomains. Nonequilibrium nanostructures can be obtained by processes, like selective solvent annealing. ${ }^{11,16-18}$ For BCPs with volume fractions near the phase boundary between two morphologies, thermally induced order-to-order transition (OOT) may also occur since the Flory-Huggins interaction parameter, $\chi$, varies as the temperature changes. ${ }^{19-24}$

BCP-based supramolecules are produced by attaching small molecules to the side chains of a block via hydrogen bonding, electrostatic interactions, or metal ligation. ${ }^{23,25-28}$ A range of morphologies can be obtained with one $\mathrm{BCP}$ by incorporating different volume fractions of the small molecules. For example, cylindrical and lamellar morphologies can be produced from BCPs with a spherical microdomain morphology and gyroidal morphologies from BCPs with a cylindrical microdomain morphology. ${ }^{23}$ Since the small molecules are not covalently bonded to either block, their interaction with the BCP can be weakened by heating, leading to a redistribution of the small molecule, ultimately resulting in an OOT. ${ }^{23}$ Unlike thermally induced OOTs, which are based on changes in the segmental interactions, the morphological transitions of BCP-based supramolecules are due to the changes in the volume fraction of each component, and

*Corresponding author. E-mail: tingxu@berkeley.edu. as such, a wider range of morphological transitions are accessible. In fact, up to five consecutive OOTs have been observed upon heating one BCP-based supramolecule from room temperature to $200{ }^{\circ} \mathrm{C} .{ }^{23}$

In thin films, BCP-based supramolecules assemble into structures similar to that seen in the bulk..$^{17,18,29-33}$ Supramolecular assemblies, such as lamellae-within-lamellae and cylinder-withinlamellae, can be macroscopically oriented over multiple length scales simultaneously. ${ }^{33}$ After selective removal of the small molecule, the BCPs will rearrange within the framework of the supramolecular morphology. Consequently, the resultant BCP morphologies are templated by the morphology of the supramolecule, and many novel nanostructures are made accessible that otherwise could not be obtained using the BCP alone.

In this study, 3- $n$-pentadecylphenol (PDP) was hydrogen bonded to the 4-vinylpyridine unit of a diblock copolymer, polystyrene-block-poly(4-vinylpyridine) (PS- $b$-P4VP), to form a supramolecule PS- $b$-P4VP(PDP). ${ }^{18,25,34}$ The molecular weights of PS and P4VP block are 20000 and 19000 Da, respectively, and in bulk, PS(20000)- $b$-P4VP(19000) forms a lamellar morphology. Upon adding PDP to a 1:1 stoichiometry, the volume fraction of P4VP(PDP) increases, and the supramolecule PS- $b$-P4VP(PDP $)_{1}$ forms a cylinder-within-lamellae hierarchical assembly. In thin films, the hexagonally packed PS-rich cylinders embedded in the P4VP(PDP) matrix are oriented normal to the surface. ${ }^{33}$ After selectively removing the PDPs and solvent annealing for a short period of time, PS(20000)- $b$-P4VP(19000) forms hexagonally packed polygonal cylinders oriented normal to the surface as confirmed by scanning probe microscopy (SPM) and grazing incidence small-angle X-ray scattering (GISAXS). The observed hexagonally packed polygonal morphology is a kinetically trapped, nonequilibrium morphology of the PS(20000)- $b$-P4VP(19000). Once the small molecules (PDPs) are removed, the BCPs are forced to assemble within the morphological framework of 


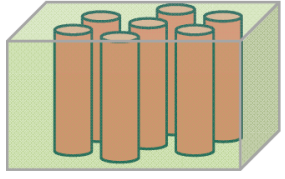

(i)

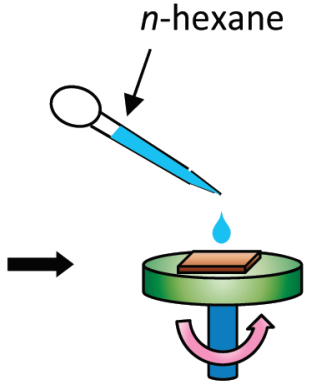

(ii)

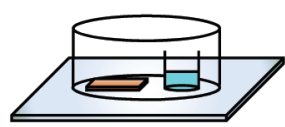

(iii)

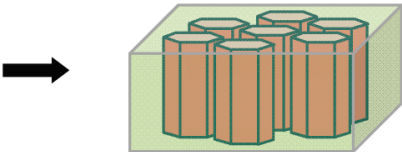

(iv)

Figure 1. Schematics of the procedure to prepare thin films with polygonal cylinders: (i) preordered PS- $b$-P4VP(PDP) supramolecular thin films, (ii) rinsed with $n$-hexane to remove most PDPs, (iii) annealed in chloroform vapor, and (iv) thin films with ordered polygonal cylinders.

the supramolecules. The BCP assembles into lamellae and locally stabilizes the facets of the polygons. Thus, the resultant hexagonally packed faceted cylinders combine both morphologies from the supramolecule and the BCP.

A large library of morphologies has been reported in the BCPbased supramolecules using di-BCPs forming sphere, cylinder, gyroid, and lamellae. This templated route to assemble the BCP within the nanostructed supramolecular frames should be applicable to other BCP-based supramolecules and may lead to novel nonequilibrium structures combining different known BCP morphologies. This approach also provides a platform to obtain nonequilibrium nanostructures and enrich our fundamental understanding on the pathway-dependent self-assembly.

\section{Experimental Section}

Diblock copolymers PS(20 000)-b-P4VP(19 000) $(\mathrm{PDI}=1.09)$ were purchased from Polymer Source, Inc. 3-n-Pentadecylphenol $(95 \%)$ was purchased from Acros. Chloroform and $n$-hexane were purchased from Fisher. All chemicals were used as received. PS- $b$-P4VP was dissolved in chloroform to form $1 \%-2 \%(\mathrm{w} / \mathrm{v})$ stock solutions. The desired amount of PDP was separately dissolved in chloroform. The PS- $b$-P4VP solution was then added dropwise to the PDP solution, followed by stirring overnight. Thin films were prepared by spin-coating the mixed solutions onto silicon wafers at spinning speed $3000 \mathrm{rpm}$. Sample thicknesses were measured using a Filmetrics F20 interferometer. For solvent annealing, thin films were placed together with a beaker of $40 \mathrm{~mL}$ of chloroform in a sealed chamber at $22^{\circ} \mathrm{C}$ for $48 \mathrm{~h}$.

Details of the reported procedure to generate polygonal cylindrical morphology in thin films are schematically shown in Figure 1 . To remove PDPs, $n$-hexane, a good solvent for PDP but a nonsolvent for PS and P4VP, was promptly dropped onto the films until the whole films were covered with $n$-hexane (the process took less than $1 \mathrm{~s}$ ), followed by immediately spinning at $3000 \mathrm{rpm}$ to spill away $n$-hexane and the dissolved PDPs. The films were then solvent-annealed again under chloroform atmosphere at $22{ }^{\circ} \mathrm{C}$ inside an inverted dish $(170 \mathrm{~mm}$ diameter $\mathrm{x}$ $90 \mathrm{~mm}$ height) on which a weight $\sim 2 \mathrm{~kg}$ was loaded.

FT-IR spectra of thin films on silicon wafer were recorded in the grazing angle attenuated total reflectance (ATR) mode on a Thermo Scientific Nicolet 6700 FT-IR, which is equipped with a Harrick GATR grazing angle ATR accessory. Thin films were pressed onto a germanium crystal during the measurements. Grazing incidence small-angle X-ray scattering (GISAXS) measurements were made at beamline 7.3.3 in the Advanced Light Source (ALS) at the Lawrence Berkeley National Laboratory (LBNL). X-ray wavelengths of $1.240 \AA$ were used, and the scattering spectra were corrected on an ADSC Quantum 4u CCD detector with an active area of $188 \mathrm{~mm}$ by $188 \mathrm{~mm}$ (2304 by 2304 pixels) and a dynamic range of 16 bit. Line-averaged intensities are reported as $I$ vs $q$, where $q=(4 \pi / \lambda) \sin (\theta / 2), \lambda$ is the wavelength of incident $\mathrm{X}$-rays, and $\theta$ is the scattering angle.
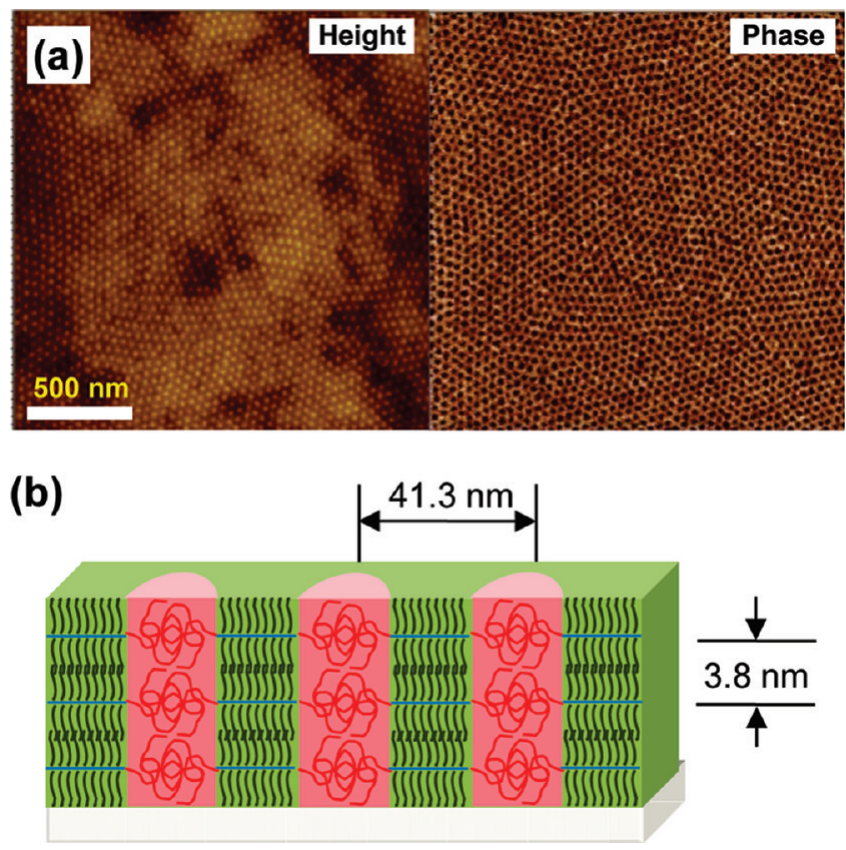

Figure 2. (a) SPM height and phase image of a PS(20 000)-b-P4VP$(19000)(P D P)_{1}$ thin film, $\sim 89 \mathrm{~nm}$ in thickness. The $z$ scale is $15 \mathrm{~nm}$ for height and $30^{\circ}$ for phase. (b) Schematic drawing of the cylinder-withinlamella hierarchical structure in thin films.

Scanning probe microscopy (SPM) images were taken on a Molecular Imaging PicoSPM II with a PicoScan 2500 controller using silicon cantilevers (PPP-NCH from Nanosensors). The spring constant of the cantilever was $10-130 \mathrm{~N} / \mathrm{m}$ with a resonant frequency in the range of $204-497 \mathrm{kHz}$. The set point for auto tune was $2.5 \mathrm{~V}$. The set-point amplitude was $90 \%$ of the free vibration value.

\section{Results and Discussion}

Figure 2a shows the SPM topography and phase images of $a \sim 89 \mathrm{~nm}$ PS(20000)- $b$-P4VP(19000)(PDP) 1 thin film after solvent annealed in a chloroform atmosphere for $48 \mathrm{~h}$. The hexagonally packed circular PS-rich domains embedded in the P4VP(PDP) matrix can be clearly seen. This was further confirmed by GISAXS study. ${ }^{33}$ Similar to what seen before, PS(20000)- $b$-P4VP(19000)$(\mathrm{PDP})_{1}$ formed hexagonally packed cylindrical microdomains (PS-rich phase) oriented normal to the surface as schematically shown in Figure 2b. The periodicity of the perpendicular PS-rich cylinders is $41.3 \mathrm{~nm}$, and the periodicity of the lamellae of the $\mathrm{P} 4 \mathrm{VP}(\mathrm{PDP})$ comb block oriented parallel to the surface is $3.8 \mathrm{~nm}$.

The PS(20000)- $b$-P4VP(19000)(PDP $)_{1}$ thin film was then rinsed using $n$-hexane, a selective solvent for PDP and a 


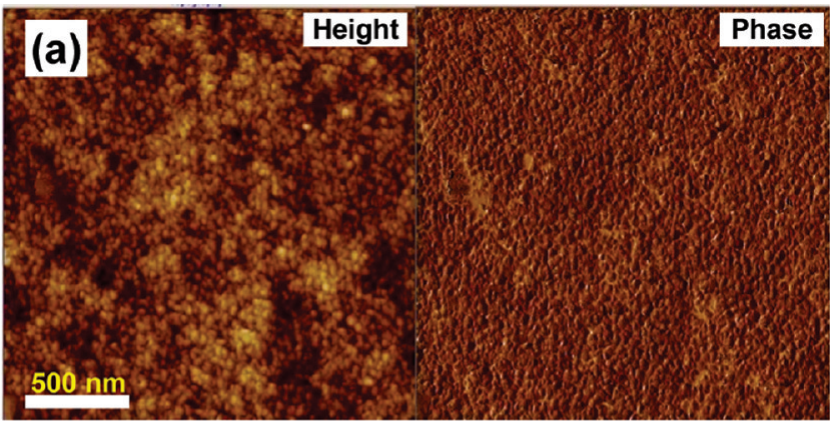

(b)

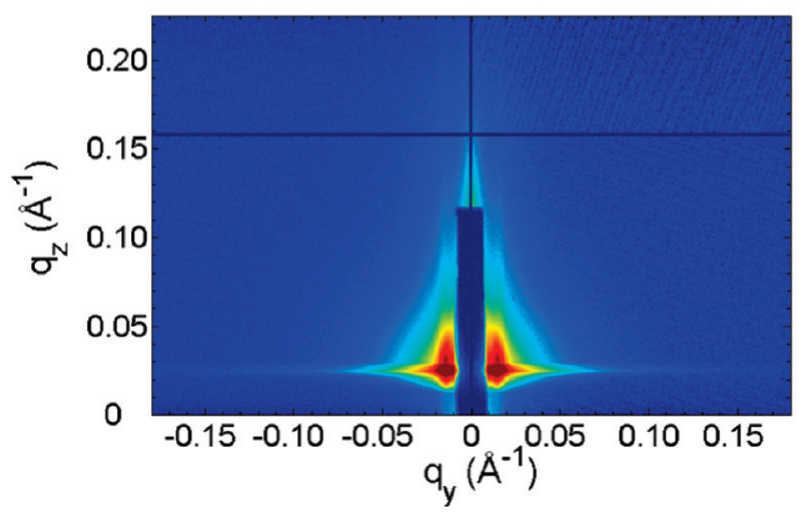

Figure 3. (a) SPM height and phase image of a $\sim 37 \mathrm{~nm}$ thin film right after rinsed with $n$-hexane. The film was originally $80 \mathrm{~nm}$ in thickness. The $z$ scale for height and phase is $30 \mathrm{~nm}$ and $90^{\circ}$. (b) GISAXS pattern of a $\sim 30 \mathrm{~nm}$ thin film right after rinsed with $n$-hexane at an incident angle of $0.2^{\circ}$. The film was originally $60 \mathrm{~nm}$ in thickness.

nonsolvent for PS and P4VP. As shown in Figure 3a, immediately following this rinse, little ordering of the film on the surface is evident. However, diffuse reflections along the $q_{y}$ and $q_{z}$ directions can still be seen in the GISAXS 2D pattern in Figure 3b, indicating that the structural framework of the initial supramolecular assembly remained. A $q_{y}$ scan confirmed that the lateral spacing of the template remained the same after the $n$-hexane rinse. Since the majority of the PDP was removed, scattering peaks along $q_{z}$ corresponding to the parallel small lamellae formed by comblike P4VP(PDP) complexes as seen in the initial PS(20 000)- $b$-P4VP(19000)(PDP $)_{1}$ thin film disappeared. ${ }^{33}$ The hexagonally packed cylindrical structure formed before rinsing with $n$-hexane is essential for the formation of polygons. A film without solvent annealing after spin coating, i.e., with lessordered cylinders, is not able to form the polygonal patterns.

Figure 4a shows the SPM topography and phase images of the $n$-hexane rinsed film that was solvent annealed for $\sim 3 \mathrm{~h}$ in chloroform vapor. The cylindrical microdomains are recovered, and more strikingly, the cross sections of the cylinders are polygonal, dominantly honeycomb-like in shape. The polygonal cylinders are more closely packed, and the edge-to-edge distance between the each cylinder is reduced from $\sim 24$ to $\sim 14.6 \mathrm{~nm}$. Figure $4 b$ shows the GISAXS 2D patterns of same thin film. Scattering peaks that only appear along $q_{y}$ axis indicate that the microdomains are oriented perpendicular to the surface. Diffraction rods along $q_{z}$ direct can still be seen, indicating the cylinders are relative uniform throughout the film. A $q_{y}$ scan at $q_{z}=$ $0.025 \AA^{-1}$, taken from the right side of the GISAXS 2D pattern, is shown in Figure 4c. The relative positions of the peaks, with respect to the first-order peak, are $1: \sqrt{ } 3: 2$, characteristic of hexagonally packed cylinders. The first-order peak at $q_{y}=$ $0.0152 \AA^{-1}$ corresponds to a spacing of $41.3 \mathrm{~nm}$, which is the same as that of the cylindrical microdomains before the $n$-hexane rinse. Although the cross sections of the hexagonal cylinders are greater than that of the circular cylinders, the center-to-center distance between the cylinders remained unchanged. With further solvent annealing $(24 \mathrm{~h})$, the polygonal cylinders gradually merged and formed the striped patterns shown in Figure 5.

The volume fractions of PS-rich cylinders in thin films were calculated based on GISAXS and the SPM images, as shown in Figure 6. Prior to the removal of the PDP, the volume fraction of the circular cylinders was $\sim 23 \%$, close to the weight fraction of PS blocks in thin films, i.e., $\sim 21 \%$ (Figure 6 a). Once the polygonal cylinders are formed, the volume fraction of the cylinders increased significantly to $\sim 48 \%$, as shown in Figure $6 \mathrm{~b}$. The hexagonally packed polygons, obtained with a lamellae-forming $\mathrm{BCP}\left(V_{\mathrm{PS}}: V_{\mathrm{P} 4 \mathrm{VP}} \sim 20000: 19000 \sim 1: 1\right)$, are unique nanostructures not seen with coil-coil symmetric BCPs. The formation of the polygonal morphology is pathway dependent and can be attributed to the templated assembly of the BCP in thin films.

$n$-Hexane is a nonsolvent for both PS and P4VP but a good solvent for PDP. After rinsing in $n$-hexane, a significant fraction of the PDP was extracted from the P4VP(PDP) domain. The SPM image shown in Figure $3 a$ indicates that the PS-rich cylinders did not remain oriented normal to the surface, and the hexagonal packing was disrupted. This can be attributed either to an orientation of the morphology during the spincoating process or from the volume change associated with the removal of the PDP. It should be noted that the film thickness decreased by $\sim 47 \%$ after the rinse with $n$-hexane, as measured using SPM and optical interferometry, and GISAXS showed that the average interdomain spacing remained constant.

During subsequent solvent annealing, the mobility of copolymer chains is enhanced by the presence of the solvent and the copolymer rearranged, leading to a recovery of ordered cylindrical structures. Locally, PS(20000)-b-P4VP(19000) forms a lamellar microdomain morphology, further stabilizing the edges of the polygonal structures. The PS domains are swollen laterally to accommodate the film thickness reduction, since there is no lateral support from the P4VP(PDP) matrix once the PDP has been removed. In addition, the PS chains in the PS- $b$-P4VP(PDP) supramolecules are deformed to accommodate the crosssectional mismatch between the coil and comb blocks at the interfaces. Once the PDP is removed, it is energetically favorable for the PS chains to rearrange laterally. Since the cylinders are originally packed hexagonally, the polygons dominantly have six facets. As discussed previously, the original circular cylindrical microdomain morphology is key to the formation of the polygonal cylinders, which means that the rearrangement and swelling of PS blocks after the rinse with $n$-hexane is constrained by the original patterns.

After rinsing with $n$-hexane, a fraction of the PDP still remained in the thin films since the time of exposure to $n$-hexane was short. On the basis of the film thickness measurements, the remaining PDP was calculated to be $\sim 10 \%$ of the original added PDP. ATR FT-IR was used to monitor the hydrogen bonding between the 4VP and PDP in the thin films. Figure 7 shows the FT-IR spectra of PS- $b$-P4VP and PS- $b$-P4VP(PDP) $)_{1}$ thin films before and after rinsing with $n$-hexane. Free pyridine groups of P4VP blocks have absorption at $993 \mathrm{~cm}^{-1}$, and upon hydrogen bonding with PDP, the band shifts to $1008 \mathrm{~cm}^{-1}$. After a single rinsing with $n$-hexane the absorption band at $993 \mathrm{~cm}^{-1}$ from the free pyridine groups was observed. A band at $1003 \mathrm{~cm}^{-1}$ is also seen, which is the superposition of the adsorptions of free and hydrogen-bonded pyridine groups, indicating that there is a trace of PDP in the thin films. However, the ATR-FTIR absorption intensity is very sensitive to the surface roughness, making it difficult to quantify the amount of PDP within the film. Methanol is a good solvent for PDP and P4VP and can disrupt the 


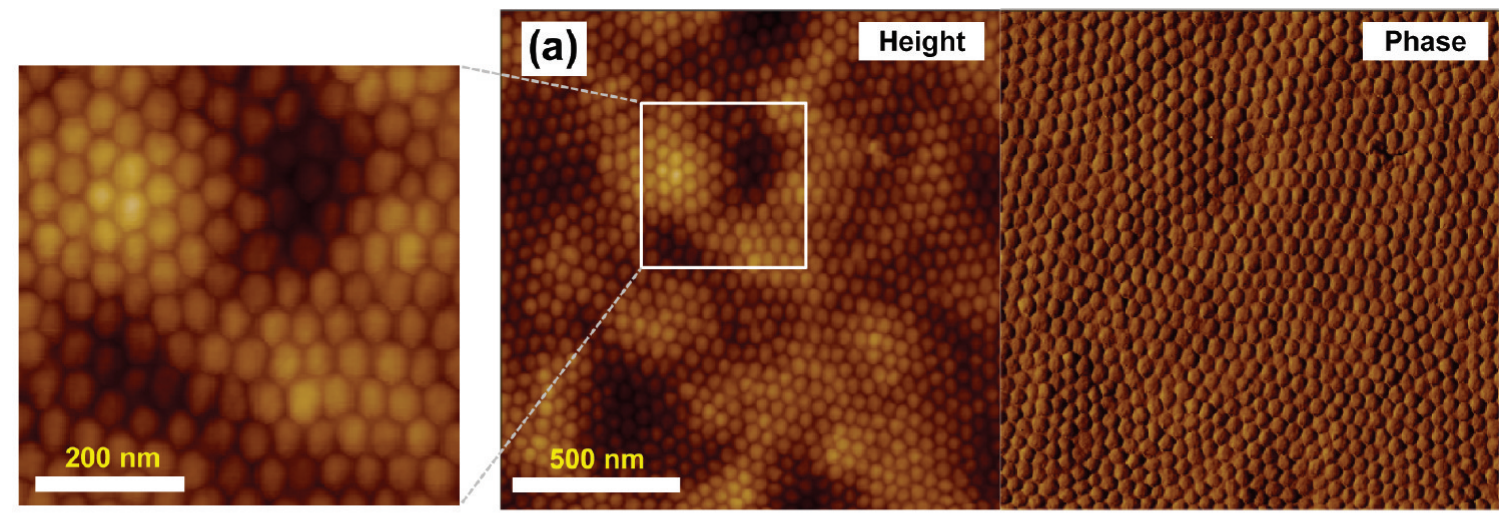

(b)



(c)

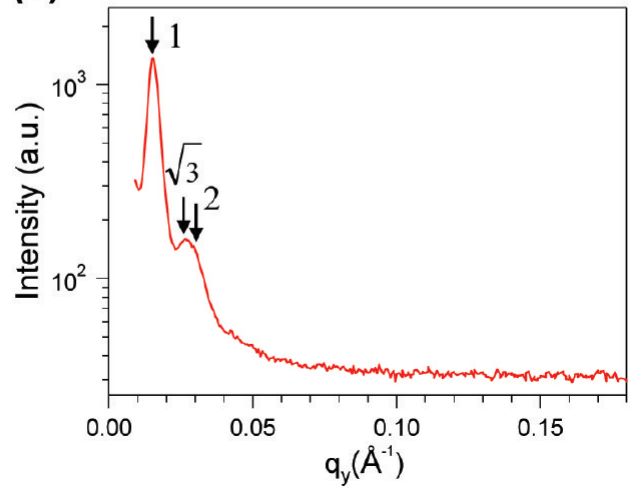

Figure 4. (a) SPM height and phase image of a $\sim 37 \mathrm{~nm}$ thin film after rinsed with $n$-hexane and annealed in chloroform vapor pressure for $3 \mathrm{~h}$. The film was originally $\sim 80 \mathrm{~nm}$ in thickness. The $z$ scale for height and phase is $20 \mathrm{~nm}$ and $80^{\circ}$. (b) GISAXS pattern of a $\sim 30 \mathrm{~nm}$ thin film after the same treatment as (a) at an incident angle of $0.2^{\circ}$. The film was originally $60 \mathrm{~nm}$ in thickness. (c) The $q_{y}$ scan at $q_{z}=0.025 \AA^{-1}$, showing the PS cylindrical microdomains are hexagonally packed and oriented perpendicular to the surface.

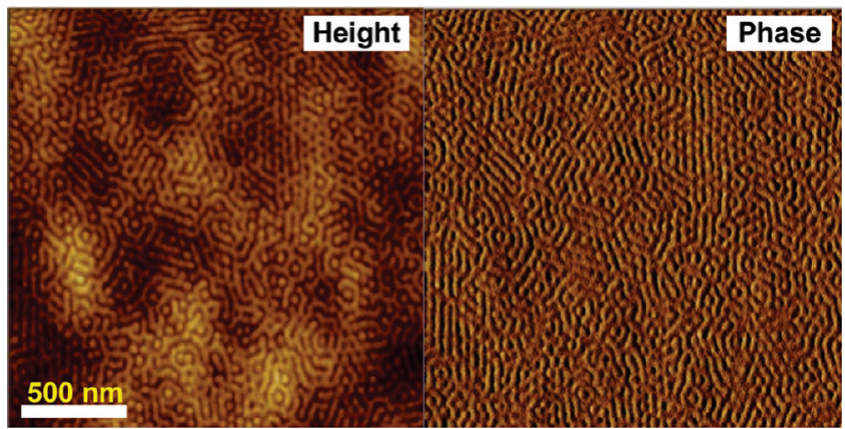

Figure 5. SPM height and phase image of a $\sim 37 \mathrm{~nm}$ thin film after rinsed with $n$-hexane and annealed in chloroform vapor pressure for $24 \mathrm{~h}$. The film was originally $\sim 80 \mathrm{~nm}$ in thickness. The $z$ scale for height and phase is $20 \mathrm{~nm}$ and $60^{\circ}$.

hydrogen bonds between PDP and P4VP. Rinsing with methanol following the same procedure completely removed the PDP, and the spectrum of the thin film is exactly the same as that of pure PS- $b$-P4VP, also shown in Figure 7.

The residual PDP plays an important role in the formation of the polygonal structures observed. Thin films washed with methanol were not able to form the polygonal patterns. Instead, the copolymer formed parallel lamellar oriented normal to the surface. The P4VP block has favorable interfacial interactions with the oxide layer on the silicon substrate and the PS has a lower surface energy than P4VP. Consequently, in thin films, the lamellar microdomains orient parallel to the (a)

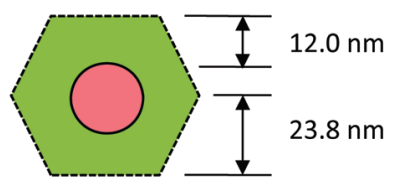

(b)

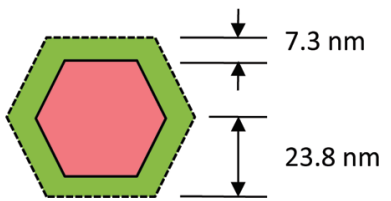

Figure 6. Surface unit cells of the ordered cylinder-in-matrix structures in thin films based on GISAXS and the SPM images: (a) hexagonally packed circular cylinders and (b) hexagonally packed hexagonal cylinders.

surface. $^{35}$ The hexagonally packed polygonal structures observed are kinetically trapped, far from the equilibrium morphology. Since these structures are oriented normal to the surface, it is evident that the PDP must mediate the interactions of the blocks with the substrate and at the surface, resulting in the perpendicular orientation. In the absence of PDP (methanol washing) the lamellar microdomains assume the expected, parallel orientation.

In summary, we presented a new approach to obtain nonequilibrium nanostructures in BCP thin films by arranging a precursor supramolecule into a morphology different from its equilibrium morphology. In particular, a BCP-based supramolecule forming cylindrical morphology was formed by 


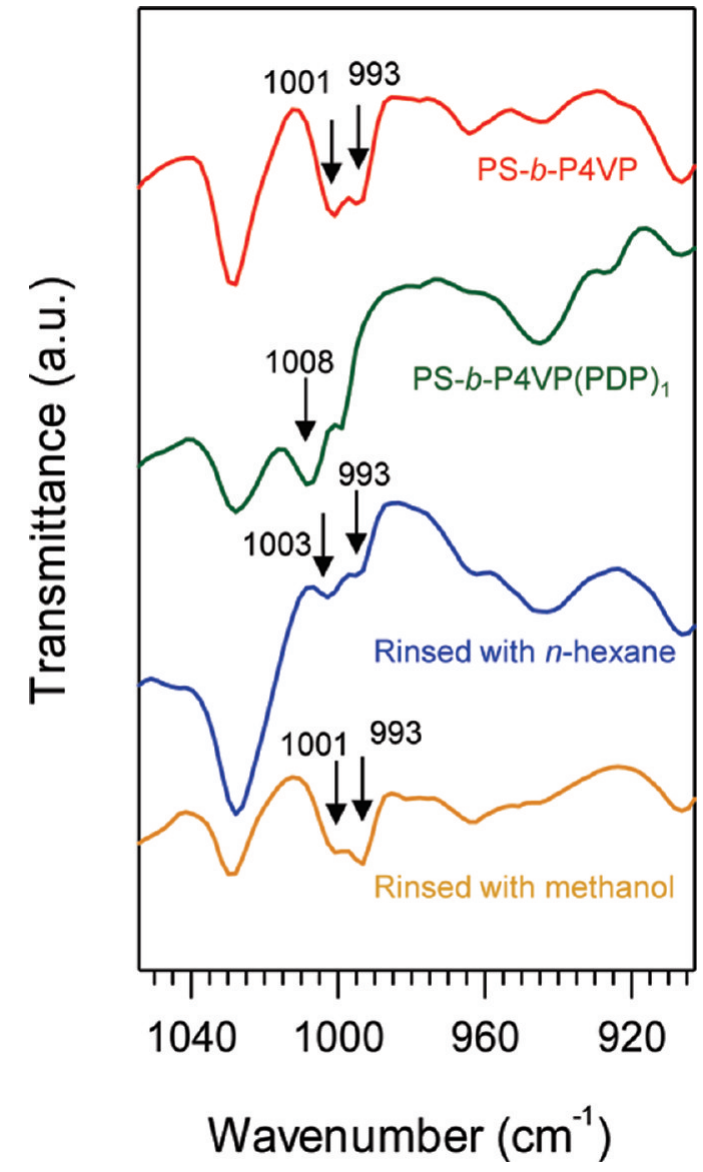

Figure 7. ATR FT-IR spectra of PS- $b$-P4VP thin films and PS$b$-P4VP(PDP $)_{1}$ thin films before and after rinsed with $n$-hexane and methanol.

hydrogen-bonding small molecules (PDP) to one block of a lamellae forming BCP. Thin films of the supramolecule were produced with hexagonally packed polygon-shaped microdomains. Upon removal of the PDP, the original block copolymer microphase separates within the boundaries established by the supramolecule forming kinetically trapped microdomain morphology templated or restrained by these boundaries. Since there are a wide range of supramolecular assemblies accessible using one single BCP, other novel morphologies can possibly be produced. This new approach also provides a platform to fundamentally understand the nonequilibrium nanostructures, especially the self-assembly processes of block copolymer in thin films.

Acknowledgment. We acknowledge Dr. Alexander Hexemer and Mr. Eliot Gann at ALS for facilitating GISAXS experiments. We thank Mr. Kris Erickson and Prof. Zettl in the Department of Physics, University of California, Berkeley, for facilitating the ATR FT-IR experiments. This work was jointly supported by the Short-term Innovative Research Grant from Army Research Office at Department of Defense under Contract W911NF07-1-0653 and the Division of Material Research, National Science Foundation (NSF-DMR), under Contract 0805301.

\section{References and Notes}

(1) Hawker, C. J.; Russell, T. P. MRS Bull. 2005, 30 (12), 952-966.

(2) Kimura, M.; Misner, M. J.; Xu, T.; Kim, S. H.; Russell, T. P. Langmuir 2003, 19 (23), 9910-9913.
(3) Thurn-Albrecht, T.; Schotter, J.; Kastle, C. A.; Emley, N.; Shibauchi, T.; Krusin-Elbaum, L.; Guarini, K.; Black, C. T.; Tuominen, M. T.; Russell, T. P. Science 2000, 290 (5499), 21262129.

(4) Park, M.; Harrison, C.; Chaikin, P. M.; Register, R. A.; Adamson, D. Science 1997, 276 (May 30), 1401.

(5) Morkved, T. L.; Lu, M.; Urbas, A. M.; Ehrichs, E. E.; Jaeger, H. M.; Mansky, P.; Russell, T. P. Science 1996, 273 (5277), 931-933.

(6) Bita, I.; Yang, J. K. W.; Jung, Y. S.; Ross, C. A.; Thomas, E. L.; Berggren, K. K. Science 2008, 321 (5891), 939-943.

(7) Tang, C. B.; Lennon, E. M.; Fredrickson, G. H.; Kramer, E. J.; Hawker, C. J. Science 2008, 322 (5900), 429-432.

(8) Ruiz, R.; Kang, H. M.; Detcheverry, F. A.; Dobisz, E.; Kercher, D. S.; Albrecht, T. R.; de Pablo, J. J.; Nealey, P. F. Science 2008, 321 (5891), 936-939.

(9) Angelescu, D. E.; Waller, J. H.; Register, R. A.; Chaikin, P. M. Adv. Mater. 2005, 17 (15), 1878-1881.

(10) Segalman, R. A.; Yokoyama, H.; Kramer, E. J. Adv. Mater. 2001, $13(15), 1152-1155$.

(11) Kim, S. H.; Misner, M. J.; Xu, T.; Kimura, M.; Russell, T. P. Adv. Mater. 2004, 16 (3), 226-231.

(12) Huang, E.; Rockford, L.; Russell, T. P.; Hawker, C. J. Nature (London) 1998, 395 (6704), 757-758.

(13) Fasolka, M. J.; Mayes, A. M. Annu. Rev. Mater. Res. 2001, 31, $323-$ 355.

(14) Lambooy, P.; Russell, T. P.; Kellogg, G. J.; Mayes, A. M.; Gallagher, P. D.; Satija, S. K. Phys. Rev. Lett. 1994, 72 (18), 2899-2902.

(15) Cavicchi, K. A.; Berthiaume, K. J.; Russell, T. P. Polymer 2005, 46 (25), 11635-11639.

(16) Kim, G.; Libera, M. Macromolecules 1998, 31 (8), 2670-2672.

(17) Sidorenko, A.; Tokarev, I.; Minko, S.; Stamm, M. J. Am. Chem. Soc. 2003, 125 (40), 12211-12216.

(18) van Zoelen, W.; Asumaa, T.; Ruokolainen, J.; Ikkala, O.; ten Brinke, G. Macromolecules 2008, 41 (9), 3199-3208.

(19) Almdal, K.; Koppi, K. A.; Bates, F. S.; Mortensen, K. Macromolecules 1992, 25 (6), 1743-1751.

(20) Vigild, M. E.; Almdal, K.; Mortensen, K.; Hamley, I. W.; Fairclough, J. P. A.; Ryan, A. J. Macromolecules 1998, 31 (17), 57025716.

(21) Ryu, C. Y.; Vigild, M. E.; Lodge, T. P. Phys. Rev. Lett. 1998, 81 (24), 5354-5357.

(22) Cho, B. K.; Jain, A.; Gruner, S. M.; Wiesner, U. Science 2004, 305 (5690), 1598-1601.

(23) Valkama, S.; Ruotsalainen, T.; Nykanen, A.; Laiho, A.; Kosonen, H.; ten Brinke, G.; Ikkala, O.; Ruokolainen, J. Macromolecules 2006, 39 (26), 9327-9336.

(24) La, Y. H.; Edwards, E. W.; Park, S. M.; Nealey, P. F. Nano Lett. 2005, 5 (7), 1379-1384.

(25) Ruokolainen, J.; Saariaho, M.; Ikkala, O.; ten Brinke, G.; Thomas, E. L.; Torkkeli, M.; Serimaa, R. Macromolecules 1999, 32 (4), $1152-1158$

(26) Ikkala, O.; ten Brinke, G. Chem. Commun. 2004, 19, 2131-2137.

(27) Osuji, C. O.; Chao, C. Y.; Ober, C. K.; Thomas, E. L. Macromolecules 2006, 39 (9), 3114-3117.

(28) Osuji, C.; Ferreira, P. J.; Mao, G. P.; Ober, C. K.; Vander Sande, J. B.; Thomas, E. L. Macromolecules 2004, 37 (26), 9903-9908.

(29) Tokarev, I.; Krenek, R.; Burkov, Y.; Schmeisser, D.; Sidorenko, A.; Minko, S.; Stamm, M. Macromolecules 2005, 38 (2), 507-516.

(30) Laforgue, A.; Bazuin, C. G.; Prud'homme, R. E. Macromolecules 2006, 39 (19), 6473-6482.

(31) Albrecht, K.; Mourran, A.; Zhu, X.; Markkula, T.; Groll, J.; Beginn, U.; deJeu, W. H.; Moeller, M. Macromolecules 2008, 41 (5), 1728-1738

(32) van Zoelen, W.; Polushkin, E.; ten Brinke, G. Macromolecules 2008, 41 (22), 8807-8814.

(33) Tung, S. H.; Kalarickal, N. C.; Mays, J. W.; Xu, T. Macromolecules 2008, 41 (17), 6453-6462.

(34) Ruokolainen, J.; ten Brinke, G.; Ikkala, O. Adv. Mater. 1999, 11 (9), 777-780.

(35) Liu, Y.; Zhao, W.; Zheng, X.; King, A.; Singh, A.; Rafailovich, M. H.; Sokolov, J.; Dai, K. H.; Kramer, E. J.; Schwarz, S. A.; Gebizlioglu, O.; Sinha, S. K. Macromolecules 1994, 27 (14), 4000-4010. 